\title{
Direct Observation of Electrolyte Degradation Mechanisms in Li-Ion Batteries
}

\author{
B. Layla Mehdi ${ }^{1}$, Patricia Abellan ${ }^{1}$, Lucas R. Parent ${ }^{1}, \mathrm{Meng} \mathrm{Gu}^{2}$, Chiwoo Park ${ }^{3}$, Wu Xu, ${ }^{4}$ Yaohui \\ Zhang, ${ }^{4,5}$ Ilke Arslan, ${ }^{4}$ Ji-Guang Zhang, ${ }^{4}$ Chong-Min Wang, ${ }^{2}$ James E. Evans, ${ }^{2}$ Nigel D. Browning ${ }^{1}$ \\ ${ }^{1}$ Fundamental and Computational Sciences Directorate, Pacific Northwest National Laboratory, \\ Richland, WA, USA. \\ 2.Environmental Molecular Science Laboratory, Pacific Northwest National Laboratory, Richland, WA, \\ USA \\ ${ }^{3}$ Department of Industrial and Manufacturing Engineering, Florida State University, Tallahassee, FL, \\ USA \\ ${ }^{4}$ Energy and Environmental Directorate, Pacific Northwest National Laboratory, Richland, WA, USA. \\ ${ }^{5 .}$ Center for Condensed Matter Science and Technology, Department of Physics, Harbin Institute of \\ Technology, PR China.
}

The rapidly growing field of new energy storage devices presents a need for non-invasive in-situ/in-operando characterization techniques, which can provide information about the fundamental processes that occur at the electrolyte/electrode interface during battery operation. The electrochemical stability of electrolytes in the new generation of high energy densities batteries is disturbed by both the oxidizing nature of the cathode and reducing nature of the anode, resulting often in the accumulation of insulating side products which leads to capacity fading. The stability of Li-ion electrolytes can be quantified by an electrochemical window that defines the specific voltage range and decomposition limits of each system. Development of in-situ liquid electrochemical stages for (scanning) transmission electron microscopes (in-situ liquid ec-(S)TEM) enables a real-time study of the electrochemical processes and provides a direct characterization of the degradation processes occurring within Li-ion battery electrolytes and at the electrolyte/electrode interface during charge/discharge cycles.

Here, we demonstrate the application of an in-situ liquid ec-(S)TEM stage to study electrolyte degradation mechanisms and new electrochemical windows for state-of-the-art Li-ion battery systems. The electrolyte breakdown is initiated by a localized interaction of the electron beam, and the resulting formation of decomposition products for five different electrolytes commonly used in $\mathrm{Li}$-ion and $\mathrm{Li}-\mathrm{O}_{2}$ battery systems have been determined $[1,2]$. Figure 1 shows two different degradation mechanisms of lithium hexafluoroarsenate $\left(\mathrm{LiAsF}_{6}\right)$ salt in 1,3-dioxolane, DOL (Figure 1a) and dimethyl carbonate, DMC (Figure 1b). The $\mathrm{LiAsF}_{6}$ salt is known to undergo reduction during the electrochemical reaction at carbonaceous anodes, forming the $\mathrm{LiF}$ precipitants that are frequently observed at the electrode surfaces after battery cycling in electrolytes containing fluorinated salts (such as $\mathrm{LiAsF}_{6}$ or $\mathrm{LiPF}_{6}$ [3]). The presence of the liquid in the in-situ liquid ec-(S)TEM cell (i.e confirmation that it was a genuine liquid process) was verified by low-loss Electron Energy Loss Spectra (EELS), as shown in Figure 1c. These results demonstrate that the electron beam has the potential to mimic electrochemical reduction, and must be taken into account during in-situ ec-STEM measurements [4].

\section{References:}

[1] K. Xu, K. Chem Rev., 104 (2004), p. 4303.

[2] E. Nasybulin, W. Xu, M. H. Engelhard, Z. M. Nie, S. D. Burton, L. Cosimbescu, M. E. Gross, J. G. Zhang, J Phys Chem C, 117 (2013), p. 2635.

[3] Verma, P., Maire, P., Novak, P. Electrochim Acta 55 (2010), p. 6332. 
[4] The research described in this paper is part of the Chemical Imaging Initiative; it was conducted under the Laboratory Directed Research and Development Program at PNNL, a multiprogram national laboratory operated by Battelle for the U.S. Department of Energy under Contract

DE-AC05-76RL01830. The electrolytes used in this work were prepared with the support from Joint Center for Energy Storage Research, an Energy Innovation Hub funded by the U.S. Department of Energy (DOE), Office of Science, Basic Energy Sciences. A portion of the research was performed using EMSL, a national scientific user facility sponsored by the Department of Energy's Office of Biological and Environmental Research and located at Pacific Northwest National Laboratory.

Development of in-situ stages was supported by DOE NNSA-SSAA grant number

DE-FG52-06NA26213, and DOE BES grant number DE-FG02-03ER46057. C.P. acknowledges support from the FSU COFRS Award 032968, the Ralph E. Powe Junior Faculty Enhancement Award, and NSF-CMMI-1334012


Figure 1. BF STEM images showing degradation in Li-ion battery electrolytes induced by the electron beam. The time evolution of degradation in $1 \mathrm{M} \mathrm{LiAsF}_{6}$ salt for two different aprotic solvents; (a) DOL and (b) DMC with the dose values per image $\left(13.7 \mathrm{e}^{-} / \mathrm{nm}^{2} \mathrm{f}\right)$ and exposure times of $\mathrm{t}=3.2 \mathrm{~s}, 60 \mathrm{~s}, 131 \mathrm{~s}$. (c) EEL spectra of 1M LiTf salt in DMSO electrolyte (purple) and 1M LiAsF6 salt in EC:DMC (green), DMC (red) and DOL (black) electrolytes measured after the in-situ liquid STEM experiments with the thickness as a function of the mean free path $(\lambda)$ in each spectrum. 